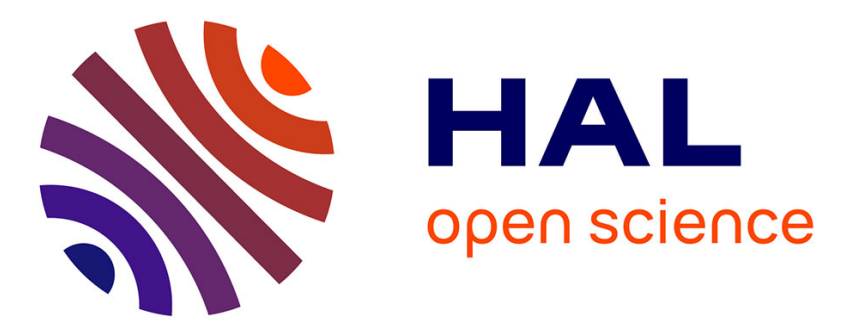

\title{
An improved pseudo-state estimator for a class of commensurate fractional order linear systems based on fractional order modulating functions
}

\author{
Yan-Qiao Wei, Da-Yan Liu, Driss Boutat, Yi-Ming Chen
}

\section{To cite this version:}

Yan-Qiao Wei, Da-Yan Liu, Driss Boutat, Yi-Ming Chen. An improved pseudo-state estimator for a class of commensurate fractional order linear systems based on fractional order modulating functions. Systems and Control Letters, 2018, 118, pp.29-34. 10.1016/j.sysconle.2018.05.011 . hal-02884211

\section{HAL Id: hal-02884211 \\ https://hal.science/hal-02884211}

Submitted on 29 Jun 2020

HAL is a multi-disciplinary open access archive for the deposit and dissemination of scientific research documents, whether they are published or not. The documents may come from teaching and research institutions in France or abroad, or from public or private research centers.
L'archive ouverte pluridisciplinaire HAL, est destinée au dépôt et à la diffusion de documents scientifiques de niveau recherche, publiés ou non, émanant des établissements d'enseignement et de recherche français ou étrangers, des laboratoires publics ou privés. 


\title{
An improved pseudo-state estimator for a class of commensurate fractional order linear systems based on fractional order modulating functions *
}

\author{
Yan-Qiao Wei ${ }^{\text {a,b }}$ Da-Yan Liu ${ }^{\text {a,b }}$ Driss Boutat ${ }^{b}$ Yi-Ming Chen ${ }^{\text {a,c,d }}$

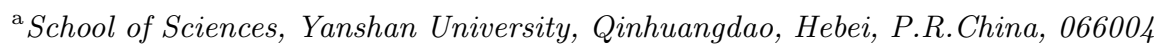 \\ ${ }^{\mathrm{b}}$ INSA Centre Val de Loire, Université d'Orléans, PRISME EA 4229, Bourges Cedex 18022, France \\ ${ }^{\mathrm{c}}$ LE STUDIUM RESEARCH PROFESSOR, Loire Valley Institute for Advanced Studies, Orléans, France \\ ${ }^{\mathrm{d}}$ PRISME (INSA-Institut National des Sciences Appliquées)-88, Boulvevard Lahitolle, 18000 Bourges, France
}

\begin{abstract}
In this paper, a non-asymptotic pseudo-state estimator for a class of commensurate fractional order linear systems is designed in noisy environment. Different from existing modulating functions methods, the proposed method is based on the system model with fractional sequential derivatives by introducing fractional order modulating functions. By applying the fractional order integration by parts formula and thanks to the properties of the fractional order modulating functions, a set of fractional derivatives and fractional order initial values of the output are analogously obtained by algebraic integral formulas. Then, an explicit formula of the pseudo-state is accomplished by using the fractional sequential derivatives of the output computed based on the previous results. This formula does not contain any source of errors in continuous noise-free case, and can be used to non-asymptotically estimate the pseudo-state in discrete noisy case. The construction of the fractional order modulating functions is also shown, which is independent of the time. Finally, simulations and comparison results demonstrate the efficiency and robustness of the proposed method.
\end{abstract}

Key words: Non-asymptotic method, Fractional order modulating functions, Robust estimation, Pseudo-state estimator, Fractional derivative initial values.

\section{Introduction}

During recent decades, the researches upon fractional order systems and controllers have been hot topics, and numerous scientific works have been proposed in various aspects [1-5]. Due to the non-locality of fractional operators, the real-state of a fractional order system can be divided into two parts: the pseudo-state and an initialization function [6]. However, the knowledge of the

\footnotetext{
* This paper was not presented at any IFAC meeting. It is under the financial support of the China Scholarship Council in China and the LE STUDIUM RESEARCH PROFESSORSHIP award of Centre-Val de Loire region in France. Corresponding author D.Y. Liu. Tel. +33-0248484091. Fax $+33-0248484045$.

Email addresses: yanqiao.wei@insa-cvl.fr (Yan-Qiao Wei), dayan.liu@insa-cvl.fr (Da-Yan Liu), driss.boutat@insa-cvl.fr (Driss Boutat), chenym@ysu.edu.cn (Yi-Ming Chen).
}

pseudo-state is quite vital in some applications of fractional order systems [6]. Thus, the estimation of the pseudo-state is obviously valuable. Among the previous works, the fractional order observers [7] and the modulating functions method [8] are notable. The fractional order observers are usually asymptotic and cannot meet some requirements of online applications $[9,10]$. To overcome this drawback, the modulating functions method has been proposed in [8] as a kind of non-asymptotic method, which is also robust against corrupting noises.

Recall that the modulating functions method was firstly introduced for linear identification in [11] and has been widely used for linear and non-linear identification of integer order systems [12]. Recently, this method has been extended to fractional order case, such as parameter estimation [13,14], pseudo-state estimation [8] and fractional order differentiators $[15,16]$. In [8], generalized modulating functions were used to derive algebraic formula for the pseudo-state by eliminating undesired 
terms. Thanks to the algebraic integrals performing as low-pass filters [17], the method was efficiently and robustly executed without knowing the initial conditions. Though, there are still a wide space remaining for further innovation. Actually, in [8] the pseudo-state was estimated by separately estimating the fractional derivatives of the output and some fractional order initial values. For this purpose, a set of fractional order differential equations were constructed, which produced more undesired terms. Thus, some more conditions were imposed to the modulating functions.

Bearing these points in mind, compared to [8], a distinctive modulating functions method is provided in this paper by introducing fractional order modulating functions. Similar to [8], the proposed estimator is also nonasymptotic and robust against noises. Superior to [8], the estimation process is greatly simplified and the computation effort is reduced with the main advantages outlined as follows.

- There is no need to construct additional equations.

- No more additional fractional order initial values are generated.

- The required fractional derivatives and fractional order initial values are analogously estimated.

- The construction of the used modulating functions is independent of time.

- Better robustness with respect to noises than [8] is shown in numerical results.

In the rest parts, the content of this paper is organized as follows. Preliminaries and the problem formulation are provided in Section 2. The advantages and the details of the proposed pseudo-state estimator are presented in Section 3, as well as the construction of the fractional order modulating functions. Numerical example and conclusions are given in the last two sections.

\section{Preliminaries}

\subsection{Fractional calculus}

This subsection presents some definitions and properties on fractional calculus, which are useful in this work.

Through this paper, the following notations are used: $I=[0, h] \subset \mathbb{R}, \alpha \in \mathbb{R}_{+}{ }^{1}$, and $l=\lceil\alpha\rceil$, where $\lceil\alpha\rceil$ denotes the smallest integer greater than or equal to $\alpha$. Then, the following definitions can be found in $[18,19]$.

Definition 1 The Riemann-Liouville fractional integral

\footnotetext{
${ }^{1}$ In this paper, $\mathbb{R}_{+}$denotes the set of positive real numbers, and $\mathbb{N}^{*}$ denotes the set of positive integers.
}

of a function $f$ is defined as follows:

$$
\left\{\begin{aligned}
\mathrm{D}_{t}^{0} f(t) & :=f(t) \\
\mathrm{D}_{t}^{-\alpha} f(t) & :=\frac{1}{\Gamma(\alpha)} \int_{0}^{t}(t-\tau)^{\alpha-1} f(\tau) d \tau,
\end{aligned}\right.
$$

where $\Gamma(\cdot)$ is the well-known Gamma function [20].

Definition 2 The Riemann-Liouville fractional derivative of a function $f$ is defined as $\mathrm{D}_{t}^{\alpha} f(t):=\frac{d^{l}}{d t^{l}}\left\{\mathrm{D}_{t}^{\alpha-l} f(t)\right\}$.

Definition 3 Let $k \in \mathbb{N}$, the Riemann-Liouville fractional sequential derivative of a function $f$ is defined as follows:

$$
{ }_{s} \mathcal{D}_{t}^{k \alpha} f(t):= \begin{cases}f(t), & \text { for } k=0, \\ D_{t}^{\alpha}\left\{{ }_{s} \mathcal{D}_{t}^{(k-1) \alpha} f(t)\right\}, & \text { for } k \geq 1 .\end{cases}
$$

Based on the additive index law of fractional derivatives [18], the following important lemma can be given.

Lemma 1 [8] The Riemann-Liouville fractional integral and derivative of a fractional sequential derivative can be given as follows: $\forall \beta \in \mathbb{R}, \forall k \in \mathbb{N}^{*}$,

$$
\mathrm{D}_{t}^{\beta}\left\{{ }_{s} \mathcal{D}_{t}^{k \alpha} f(t)\right\}=\mathrm{D}_{t}^{\beta+k \alpha} f(t)-\phi_{\beta, k, \alpha}\{f(t)\},
$$

where

$$
\phi_{\beta, k, \alpha}\{f(t)\}:=\sum_{j=1}^{k} \psi_{\beta+(j-1) \alpha, \alpha}\left\{{ }_{s} \mathcal{D}_{t}^{(k-j) \alpha} f(t)\right\} .
$$

$\psi_{\beta, \alpha}\{f(t)\}$ is a decreasing function of $t$ defined by:

$$
\psi_{\beta, \alpha}\{f(t)\}:=\sum_{i=1}^{\lceil\alpha\rceil} c_{\beta, i} t^{-\beta-i}\left[D_{t}^{\alpha-i} f(t)\right]_{t=0}
$$

with

$$
c_{\beta, i}=\left\{\begin{array}{cl}
0, & \text { if } \beta \in \mathbb{Z} \\
\frac{1}{\Gamma(1-\beta-i)}, & \text { else. }
\end{array}\right.
$$

Moreover, we have: $\forall k \geq 2$,

$$
{ }_{s} \mathcal{D}_{t}^{k \alpha} f(t)=\mathrm{D}_{t}^{k \alpha} f(t)-\phi_{\alpha,(k-1), \alpha}\{f(t)\} .
$$

Consequently, (7) provides the relationship between the derivatives introduced in Definition 2 and Definition 3. Let us introduce some other kinds of fractional derivatives with useful formulas. 
Definition 4 [19] The right-sided Caputo fractional derivative of a function $f$ is defined on $[0, h[$ as follows:

$$
\left\{\begin{aligned}
{ }^{C} \mathrm{D}_{t, h}^{0} f(t): & =f(t), \\
{ }^{C} \mathrm{D}_{t, h}^{\alpha} f(t): & =\frac{(-1)^{l}}{\Gamma(l-\alpha)} \int_{t}^{h}(\tau-t)^{l-\alpha-1} f^{(l)}(\tau) d \tau .
\end{aligned}\right.
$$

The fractional order integration by parts formula is an indispensable tool in this work, which is given in the following lemma.

Lemma 2 [21] For any interval $[0, t] \subset I$, the following formula holds:

$$
\begin{aligned}
\int_{0}^{t} g(\tau) & \mathrm{D}_{\tau}^{\alpha} f(\tau) d \tau=\int_{0}^{t}{ }^{C} \mathrm{D}_{\tau, t}^{\alpha} g(\tau) f(\tau) d \tau \\
+ & \sum_{k=0}^{\lceil\alpha\rceil-1}(-1)^{k}\left[g^{(k)}(\tau) \mathrm{D}_{\tau}^{\alpha-1-k} f(\tau)\right]_{\tau=0}^{\tau=t}
\end{aligned}
$$

Finally, the following fractional derivative will be quite important in the sequel.

Definition 5 The right-sided Caputo fractional sequential derivative of a function $f$ is defined as follows:

$$
{ }_{s}^{C} \mathcal{D}_{t, h}^{k \alpha} f(t):= \begin{cases}f(t), & \text { for } k=0 \\ { }^{C} \mathrm{D}_{t, h}^{\alpha}\left\{{ }_{s}^{C} \mathcal{D}_{t, h}^{(k-1) \alpha} f(t)\right\}, & \text { for } k \geq 1 .\end{cases}
$$

Remark that several kinds of fractional derivatives are previously defined for different purposes. Firstly, the considered fractional order systems are defined based on the Riemann-Liouville fractional derivatives. Secondly, the pseudo-state will be expressed by the fractional sequential derivatives of the output. Thanks to Lemma 1, the latter will be obtained by calculating the RiemannLiouville fractional derivatives and some fractional order initial values of the output. Finally, instead of calculating directly the Riemann-Liouville fractional derivatives of the output, algebraic integral formulas will be provided by applying Lemma 2, where the right-sided Caputo fractional derivatives of constructed modulating functions need to be explicitly calculated.

\subsection{Problem formulation}

Let us consider the following commensurate fractional order linear system within this framework:

$$
\begin{aligned}
\mathrm{D}_{t}^{\alpha} x & =A x+B u, \\
y & =C x
\end{aligned}
$$

on $I \subset \mathbb{R}_{+} \cup\{0\}$, where $A \in \mathbb{R}^{N \times N}, B \in \mathbb{R}^{N \times 1}, C \in$ $\mathbb{R}^{N \times 1}, \mathrm{D}_{t}^{\alpha} x=\left(\mathrm{D}_{t}^{\alpha} x_{1}, \ldots, \mathrm{D}_{t}^{\alpha} x_{N}\right)^{T}$ with $\alpha=\frac{1}{q}, q, N \in$ $\mathbb{N}^{*}, x \in \mathbb{R}^{N}$ is the pseudo-state column vector with unknown initial values, $y \in \mathbb{R}$ and $u \in \mathbb{R}$ are the output and the input, respectively. It is assumed $0<\alpha<2$ to guarantee the stability [22]. Moreover, the following conditions are assumed to be fulfilled:

$$
\begin{aligned}
& \left(C_{1}\right): \operatorname{rank}\left(C^{T} \quad A^{T} C^{T} \quad \cdots\left(A^{T}\right)^{N-1} C^{T}\right)^{T}=N, \\
& \left(C_{2}\right): C B=C A B=\cdots=C A^{N-2} B=0, \\
& \left(C_{3}\right): C A^{N-1} B \neq 0 .
\end{aligned}
$$

This paper aims at estimating the pseudo-state $x$ in noisy environment. To achieve the objective, by applying the modulating functions method, the system defined by (11)-(12) firstly needs to be transformed into a fractional order differential equation as done in [8].

Lemma 3 [8] If the system defined by (11)-(12) satisfies conditions $\left(C_{1}\right)-\left(C_{3}\right)$, then it can be written into the following form on $I$ :

$$
\sum_{i=0}^{N} a_{i s} \mathcal{D}_{t}^{\alpha_{i}} y(t)=\bar{u}(t)
$$

where $\bar{u}=C A^{N-1} B u, \alpha_{i}=i \alpha$, for $i=0, \ldots, N, a_{N}=$ $1, a_{i} \in \mathbb{R}$, for $i=0, \ldots, N-1$, are coefficients of the characteristic polynomial of $A$. Moreover, the pseudostate variables of $x$ can be written as linear combinations of the fractional sequential derivatives of the output $y$ :

$$
x=M_{2}^{-1} M_{1}\left(y,{ }_{s} \mathcal{D}_{t}^{\alpha} y, \cdots,{ }_{s} \mathcal{D}_{t}^{(N-1) \alpha} y\right)^{T},
$$

$$
\begin{gathered}
\text { where } M_{1}=\left(\begin{array}{cccccc}
a_{1} & a_{2} & a_{3} & \cdots & a_{N-1} & 1 \\
\vdots & \vdots & \vdots & \ddots & \vdots & \vdots \\
a_{N-1} & 1 & 0 & \cdots & 0 & 0 \\
1 & 0 & 0 & \cdots & 0 & 0
\end{array}\right) \text {, and } \\
M_{2}=\left(\begin{array}{c}
C A^{N-1}+\sum_{i=1}^{N-1} a_{i} C A^{i-1} \\
\vdots \\
C A^{N-j}+\sum_{i=j}^{N-1} a_{i} C A^{i-j} \\
\vdots \\
C
\end{array}\right.
\end{gathered}
$$

\section{Main results}

In this section, the fractional sequential derivatives ${ }_{s} \mathcal{D}_{t}^{k \alpha} y$ for $k=0, \ldots, N-1$, will be calculated by means 
of fractional derivatives and a set of fractional order initial values which are expressed by algebraic integral formulas. Thus, the pseudo-state $x$ can be given by explicit integral formulas using (14). To achieve this objective, the previously mentioned fractional order modulating functions are introduced as follows.

Definition 6 Let $\bar{I}=[\bar{a}, \bar{b}] \subset \mathbb{R}, N \in \mathbb{N}^{*}, j \in$ $\{0, \ldots, N-1\}$, and $g_{j}$ be a function satisfying the following property: for $j^{\prime}=0, \ldots, N$,

$\left(P_{5}\right):{ }_{s}^{C} \mathcal{D}_{\tau, t}^{j^{\prime} \alpha}\left\{g_{j}\right\}$ exists and is integrable on $\bar{I}$.

- If the two following properties are fulfilled: for $j^{\prime}=$ $0, \ldots, N-1$,

$\left(P_{6}^{l}\right):\left[{ }_{s}^{C} \mathcal{D}_{\tau, t}^{j^{\prime} \alpha}\left\{g_{j}(\tau)\right\}\right]_{\tau=\bar{a}}=0$,

$\left(P_{7}^{l}\right):\left[{ }_{s}^{C} \mathcal{D}_{\tau, t}^{j^{\prime} \alpha}\left\{g_{j}(\tau)\right\}\right]_{\tau=\bar{b}}=\delta_{j^{\prime} j}$

where $\delta_{k n}$ is the Kronecker delta function defined as follows [23]: $\delta_{k n}=1$ if $k=n, \delta_{k n}=0$ else, then $g_{j}$ is called left-sided $(N, j)^{\text {th }}$ order fractional modulating function on $\bar{I}$.

- If the two following properties are fulfilled: for $j^{\prime}=$ $0, \ldots, N-1$,

$\left(P_{6}^{r}\right):\left[{ }_{s}^{C} \mathcal{D}_{\tau, t}^{j^{\prime} \alpha}\left\{g_{j}(\tau)\right\}\right]_{\tau=\bar{a}}=\delta_{j^{\prime} j}$,
$\left(P_{7}^{r}\right):\left[{ }_{s}^{C} \mathcal{D}_{\tau, t}^{j^{\prime} \alpha}\left\{g_{j}(\tau)\right\}\right]_{\tau=\bar{b}}=0$,

then $g_{j}$ is called right-sided $(N, j)^{\text {th }}$ order fractional modulating function on $\bar{I}$.

\subsection{Explicit integral formula of the pseudo-state}

In this subsection, the main goal is to provide algebraic integral formulas for the fractional sequential derivatives ${ }_{s} \mathcal{D}_{t}^{k \alpha} y$ for $k=0, \ldots, N-1$, which are used to express the pseudo-state $x$ by using (14). According to $(7),{ }_{s} \mathcal{D}_{t}^{k \alpha} y$ can be obtained by means of the fractional derivatives $\mathrm{D}_{t}^{k \alpha} y$ and the corresponding fractional order initial values $\left[\mathrm{D}_{t}^{\alpha-1}\left\{{ }_{s} \mathcal{D}_{t}^{j \alpha} y(t)\right\}\right]_{t=0}$ for $j=0, \ldots, N-3$. Moreover, $\mathrm{D}_{t}^{k \alpha} y$ can be calculated using (3) by $\mathrm{D}_{t}^{\alpha-1}\left\{{ }_{s} \mathcal{D}_{t}^{j \alpha} y(t)\right\}$ with $j=k-(1-q)$ and the previous fractional order initial values. Consequently, we will first express $\mathrm{D}_{t}^{\alpha-1}\left\{{ }_{s} \mathcal{D}_{t}^{j \alpha} y(t)\right\}$ by algebraic integral formulas (see Theorem 1), and then in a second result we will provide algebraic integral formulas for the initial conditions $\left[\mathrm{D}_{t}^{\alpha-1}\left\{{ }_{s} \mathcal{D}_{t}^{j \alpha} y(t)\right\}\right]_{t=0}$ (see Theorem 2).

Theorem 1 Let $y$ be the output of the system defined in (11)-(12) on I. For $0<t \in I$, suppose that $g_{j}^{l}$ for $j=0, \ldots, N-1$, are functions on $[0, t]$ satisfying $\left(P_{5}\right)$ and $\left(P_{6}^{l}\right)$, where $\bar{a}=0$ and $\bar{b}=t$ in Definition 6. Then, $\mathrm{D}_{t}^{\alpha-1}\left\{{ }_{s} \mathcal{D}_{t}^{j \alpha} y(t)\right\}$, for $j=0, \ldots, N-1$ can be obtained by solving the following linear system:

$$
\Lambda^{l}(t) \Theta(t)=\Omega^{l}
$$

where $\forall \tau \in[0, t]$, the elements of $\Lambda^{l}(\tau) \in \mathbb{R}^{N \times N}, \Theta(\tau) \in$ $\mathbb{R}^{N \times 1}$ and $\Omega^{l} \in \mathbb{R}^{N \times 1}$ are defined by: for $j=0, \ldots, N-1$,

$$
\Theta_{j}(\tau)=\mathrm{D}_{\tau}^{\alpha-1}\left\{{ }_{s} \mathcal{D}_{\tau}^{j \alpha} y(\tau)\right\}
$$

$$
\Omega_{j}^{l}=\int_{0}^{t} g_{j}^{l}(\tau) \bar{u}(\tau) d \tau-\sum_{i=0}^{N} a_{i} \int_{0}^{t}{ }_{s}^{C} \mathcal{D}_{\tau, t}^{i \alpha}\left\{g_{j}^{l}(\tau)\right\} y(\tau) d \tau
$$

and for $i=1, \ldots, N, j=0, \ldots, N-1$,

$$
\Lambda_{j, i}^{l}(\tau)=\sum_{j^{\prime}=0}^{N-i} a_{i+j^{\prime} s}^{C} \mathcal{D}_{\tau, t}^{j^{\prime} \alpha}\left\{g_{j}^{l}(\tau)\right\}
$$

Moreover, if $g_{j}^{l}$ for $j=0, \ldots, N-1$, are left-sided $(N, j)^{\text {th }}$ order fractional modulating functions on $[0, t]$, then we have: $\forall j \in\{0, \ldots, N-1\}$,

$$
\mathrm{D}_{t}^{\alpha-1}\left\{{ }_{s} \mathcal{D}_{t}^{j \alpha} y(t)\right\}=\sum_{i=0}^{j} \lambda_{j-i} \Omega^{l}(N-1-i),
$$

where

$$
\lambda_{j}=\left\{\begin{array}{cl}
1, & \text { if } j=0 \\
-\sum_{i=0}^{j-1} a_{N-i-1} \lambda_{j-i-1}, & \text { else. }
\end{array}\right.
$$

Proof. The proof can be given in following steps.

Step 1: Multiplication and integration. By multiplying $N$ functions $g_{j}^{l}$ for $j=0, \ldots, N-1$ to (13) and integrating from 0 to $t$, we get:

$$
\sum_{i=0}^{N} a_{i} \int_{0}^{t} g_{j}^{l}(\tau){ }_{s} \mathcal{D}_{\tau}^{i \alpha} y(\tau) d \tau=\int_{0}^{t} g_{j}^{l}(\tau) \bar{u}(\tau) d \tau
$$

Step 2: Fractional order integration by parts. By sequently applying $N$ times fractional order integration by parts formula given in Lemma 2 to (21), we obtain: 
for $j=0, \ldots, N-1$

$$
\begin{aligned}
& \sum_{i=0}^{N} a_{i} \int_{0}^{t}{ }_{s}^{C} \mathcal{D}_{\tau, t}^{i \alpha}\left\{g_{j}^{l}(\tau)\right\} y(\tau) d \tau= \\
& \int_{0}^{t} g_{j}^{l}(\tau) \bar{u}(\tau) d \tau-\sum_{j^{\prime}=1}^{N} \sum_{i=j^{\prime}}^{N} a_{i} \\
& \times\left[{ }_{s}^{C} \mathcal{D}_{\tau, t}^{\left(j^{\prime}-1\right) \alpha}\left\{g_{j}^{l}(\tau)\right\} D_{\tau}^{\alpha-1}\left\{{ }_{s} \mathcal{D}_{\tau}^{\left(i-j^{\prime}\right) \alpha} y(\tau)\right\}\right]_{\tau=0}^{\tau=t}
\end{aligned}
$$

Step 3: Solving linear systems. By regrouping the terms in the double sum in (22), we get: for $j=0, \ldots, N-1$,

$\sum_{j^{\prime}=1}^{N} \sum_{i=j^{\prime}}^{N} a_{i}\left[{ }_{s}^{C} \mathcal{D}_{\tau, t}^{\left(j^{\prime}-1\right) \alpha}\left\{g_{j}^{l}(\tau)\right\} \mathrm{D}_{\tau}^{\alpha-1}\left\{{ }_{s} \mathcal{D}_{\tau}^{\left(i-j^{\prime}\right) \alpha} y(\tau)\right\}\right]_{\tau=0}^{\tau=t}=$
$\sum_{i=1}^{N}\left[\left(\sum_{j^{\prime}=0}^{N-i} a_{i+j^{\prime} s}{ }_{s} \mathcal{D}_{\tau, t}^{j^{\prime} \alpha}\left\{g_{j}^{l}(\tau)\right\}\right) \mathrm{D}_{\tau}^{\alpha-1}\left\{{ }_{s} \mathcal{D}_{\tau}^{(i-1) \alpha} y(\tau)\right\}\right]_{\tau=0}^{\tau=t}$

Based on the notations given in (16)-(18) and using (23), (22) can be rewritten into a matrix form: $\Lambda^{l}(t) \Theta(t)-\Lambda^{l}(0) \Theta(0)=\Omega^{l}$. Hence, if $\left(P_{6}^{l}\right)$ is fulfilled, then $\Lambda^{l}(0)$ is eliminated and (15) is obtained. Moreover, if $\left(P_{7}^{l}\right)$ is fulfilled, then $\Lambda^{l}(t)$ becomes $M_{1}$. Its inverse is given as $M_{1}^{-1}=\left(\begin{array}{ccccc}0 & 0 & \cdots & 0 & 1 \\ 0 & 0 & \cdots & 1 & \lambda_{1} \\ \vdots & \vdots & \vdots & \ddots & \vdots \\ 1 & \lambda_{1} & \cdots & \lambda_{N-2} & \lambda_{N-1}\end{array}\right)$, where $\lambda_{j}$ for $j=0, \ldots, N-1$ are defined in (20). Thus, this proof can be completed by the solution $\Theta(t)=M_{1}{ }^{-1} \Omega^{l}$ of (15).

In the following theorem, the algebraic integral formulas for $\left[\mathrm{D}_{t}^{\alpha-1}\left\{{ }_{s} \mathcal{D}_{t}^{j \alpha} y(t)\right\}\right]_{t=0}$ for $j=0, \ldots, N-1$ are given.

Theorem 2 Let $y$ be the output of the system defined in (11)-(12) on I. For $0<t \in I$, suppose that $g_{j}^{r}$ for $j=0, \ldots, N-1$, are functions on $[0, t]$ satisfying $\left(P_{5}\right)$ and $\left(P_{6}^{r}\right)$, where $\bar{a}=0$ and $\bar{b}=t$ in Definition 6 . Then, $\left[\mathrm{D}_{\tau}^{\alpha-1}\left\{{ }_{s} \mathcal{D}_{\tau}^{j \alpha} y(\tau)\right\}\right]_{\tau=0}$, for $j=0, \ldots, N-1$ can be obtained by solving the following linear system:

$$
\Lambda^{r}(0) \Theta(0)=-\Omega^{r}
$$

where $\forall \tau \in[0, t]$, the elements of $\Lambda^{r}(\tau)$ and $\Omega^{r}$ are defined by substituting $g_{j}^{l}$ by $g_{j}^{r}$ in (17) and (18) respectively, and $\Theta(0)$ is defined by taking $\tau=0$ in (16) . Moreover, if $g_{j}^{r}$ for $j=0, \ldots, N-1$, are right-sided $(N, j)^{\text {th }}$ order fractional modulating functions on $[0, t]$, then we have: $\forall j \in\{0, \ldots, N-1\}$,

$$
\left[\mathrm{D}_{\tau}^{\alpha-1}\left\{{ }_{s} \mathcal{D}_{\tau}^{j \alpha} y(\tau)\right\}\right]_{\tau=0}=-\sum_{i=0}^{j} \lambda_{j-i} \Omega^{r}(N-1-i)
$$

where $\lambda_{j-i}$ are defined in (20).

Proof. This proof is similar to the one of Theorem 1. By substituting $g_{j}^{l}$ by $g_{j}^{r}$ in (21)-(23), we can get $\Lambda^{r}(t) \Theta(t)-\Lambda^{r}(0) \Theta(0)=\Omega^{r}$. Hence, if $\left(P_{6}^{r}\right)$ is fulfilled, then $\Lambda^{r}(t)$ is eliminated and (24) is obtained. Moreover, if $\left(P_{7}^{r}\right)$ is fulfilled, then $(25)$ can be obtained.

Based on the results of Theorem 1 and Theorem 2, we are ready to give the required fractional derivatives $\mathrm{D}_{t}^{k \alpha} y$ for $k=0, \ldots, N-1$ in the following corollary.

Corollary 1 Under the same hypotheses given in Theorem $1, \mathrm{D}_{t}^{k \alpha} y$ with $\alpha=\frac{1}{q}\left(q \in \mathbb{N}^{*}\right)$ are given as follows:

- for $k=1-q, \ldots, N-q$,

$$
\mathrm{D}_{t}^{k \alpha} y(t)=\mathrm{D}_{t}^{\alpha-1}\left\{{ }_{s} \mathcal{D}_{t}^{i \alpha} y(t)\right\}+\phi_{\alpha-1, i, \alpha}\{y(t)\},
$$

with $i=k-(1-q)$,

- for $k=N+1-q, \ldots, N-1$,

$$
\mathrm{D}_{t}^{k \alpha} y(t)=\mathrm{D}_{t}^{i^{\prime} \alpha-1}\left\{{ }_{s} \mathcal{D}_{t}^{N \alpha} y(t)\right\}+\phi_{i^{\prime} \alpha-1, N, \alpha}\{y(t)\},
$$

with $i^{\prime}=k-(N-q)$, and

$$
\begin{aligned}
& \mathrm{D}_{t}^{i^{\prime} \alpha-1}\left\{{ }_{s} \mathcal{D}_{t}^{N \alpha} y(t)\right\}= \\
& \mathrm{D}_{t}^{i^{\prime} \alpha-1} \bar{u}(t)-\sum_{i=0}^{N-1} a_{i} \mathrm{D}_{t}^{i^{\prime} \alpha-1}\left\{{ }_{s} \mathcal{D}_{t}^{i \alpha} y(t)\right\}
\end{aligned}
$$

where $\mathrm{D}_{t}^{i^{\prime} \alpha-1}\left\{{ }_{s} \mathcal{D}_{t}^{i \alpha} y(t)\right\}$ for $i=0, \ldots, N-1$, are given by Theorem 1 if $i^{\prime}=1$, and by:

$$
\mathrm{D}_{t}^{i^{\prime} \alpha-1}\left\{{ }_{s} \mathcal{D}_{t}^{i \alpha} y(t)\right\}=\mathrm{D}_{t}^{k^{\prime} \alpha} y(t)-\phi_{i^{\prime} \alpha-1, i, \alpha}\{y(t)\},
$$

else, with $k^{\prime}=i+i^{\prime}-q \in\{1-q, \ldots, k-1\}$. Moreover, $\phi_{i^{\prime} \alpha-1, i, \alpha}\{y(t)\}$ for $i^{\prime}=1, \ldots, q-1, i=0, \ldots, N$, are given by using (4) and $\left[\mathrm{D}_{t}^{\alpha-1}\left\{{ }_{s} \mathcal{D}_{t}^{j \alpha} y(t)\right\}\right]_{t=0}$ for $j=$ $0, \ldots, N-1$, which are given by (25).

Proof. Firstly, (26), (27) and (29) can be obtained by applying (3). Secondly, (28) can be obtained by applying $\mathrm{D}_{t}^{i^{\prime} \alpha-1}$ to (13) for $i^{\prime}=1, \ldots, q-1$. Then, this proof can be completed using a recursive way. 
With the computation results given in Theorem 1, Theorem 2 and Corollary 1, we are ready to give the algorithm that enables us to compute the pseudo-state $x$ :

Step 1. By applying (19) and (25), compute $D_{t}^{\alpha-1}\left\{{ }_{s} \mathcal{D}_{t}^{j \alpha} y\right\}$ and $\left[\mathrm{D}_{t}^{\alpha-1}\left\{{ }_{s} \mathcal{D}_{t}^{j \alpha} y(t)\right\}\right]_{t=0}$ for $j=0, \ldots, N-1$.

Step 2. By applying (4), compute $\phi_{i^{\prime} \alpha-1, i, \alpha}\{y(t)\}$ for $i^{\prime}=1, \ldots, q-1, i=0, \ldots, N$.

Step 3. For $k=1-q, \ldots, N-q$, compute $\mathrm{D}_{t}^{k \alpha} y$ by applying (26).

Step 4. For $k=N+1-q, \ldots, N-1$, set $i^{\prime}=k-$ $(N-q)$. If $i^{\prime}>1$, then by applying (29) compute $\mathrm{D}_{t}^{i^{\prime} \alpha-1}\left\{{ }_{s} \mathcal{D}_{t}^{i \alpha} y\right\}$ for $i=0, \ldots, N-1$.

Step 5. By applying (28), compute $\mathrm{D}_{t}^{i^{\prime} \alpha-1}\left\{{ }_{s} \mathcal{D}_{t}^{N \alpha} y\right\}$.

Step 6. By applying (27), compute $\mathrm{D}_{t}^{k \alpha} y$.

Step 7. By applying Definition 3 and (7), compute ${ }_{s} \mathcal{D}_{t}^{k \alpha} y$ for $k=0, \ldots, N-1$ using $\mathrm{D}_{t}^{k \alpha} y$.

Step 8. By applying (14), compute the pseudo-state $x$ using ${ }_{s} \mathcal{D}_{t}^{k \alpha} y$.

By using this algorithm, the algebraic formulas can provide estimation with convergence in finite-time [8]. Meanwhile, the integrals can be considered as low-pass filters [17]. Thus, non-asymptotic and robust pseudostate estimator can be designed in discrete noisy case. Before doing so, the used modulating functions should be constructed.

\subsection{Construction of modulating functions}

There exist several kinds of modulating functions in the literature [24]. Since the right-sided Caputo fractional derivative of a unusual function can not be analytically calculated, polynomial type modulating functions were considered in [8] to be conveniently operated by fractional derivatives. In the proposed method, power functions are chosen to construct the required modulating functions with the expected properties. A useful formula is provided firstly in the following lemma, which can be obtained by sequently applying the property of the rightsided Caputo fractional derivative given in [19].

Lemma 4 Let $m, j^{\prime} \in \mathbb{N}$ and $0<\alpha<1$, then we have: ${ }_{s}^{C} \mathcal{D}_{\tau, t}^{j^{\prime} \alpha}(t-\tau)^{m \alpha}=$

$\left\{\begin{array}{cl}\frac{\Gamma(m \alpha+1)}{\Gamma\left(\left(m-j^{\prime}\right) \alpha+1\right)}(t-\tau)^{\left(m-j^{\prime}\right) \alpha}, & \text { if } m \geq j^{\prime}, \\ 0, & \text { else. }\end{array}\right.$

The details of the construction of modulating functions are provided in the following proposition.

Proposition 1 For $0<t \in I$, and $j \in\{0, \ldots, N-1\}$, let $g_{j}$ be a function defined on $[0, t]$ as $g_{j}(\tau)=$ $\sum_{i=0}^{N_{j}^{\prime}-1} c_{i} t^{-i \alpha}(t-\tau)^{(i+j) \alpha}$, where $N_{j}^{\prime}=2 N-j$, the coefficient $c=\left(c_{0}, c_{1}, \ldots, c_{N_{j}^{\prime}-1}\right)^{T}$ is the solution of the following linear system:

$$
Q c=d,
$$

where $d \in \mathbb{R}^{N_{j}^{\prime} \times 1}$, and $Q \in \mathbb{R}^{N_{j}^{\prime} \times N_{j}^{\prime}}$ is defined by: for $k, i=0, \ldots, N_{j}^{\prime}-1$,

$$
\begin{aligned}
& Q(k, i)= \\
& \left\{\begin{array}{cl}
\Gamma((i+j) \alpha+1), & \text { if } k \leq N-1-j \text { and } k=i, \\
\frac{\Gamma((i+j) \alpha+1)}{\Gamma((i-k+N) \alpha+1)}, & \text { if } N-j \leq k<N, \\
\frac{\Gamma((i+j) \alpha+1)}{\Gamma((i-k+N) \alpha+1)}, & \text { if } k \geq N \text { and } i \geq k-N, \\
0, & \text { else. }
\end{array}\right.
\end{aligned}
$$

- If $d$ is defined by: for $k=0, \ldots, N_{j}^{\prime}-1, d(k)=\delta_{k 0}$, then $g_{j}$ is a left-sided $(N, j)^{\text {th }}$ order fractional modulating function on $[0, t]$, i.e. $\left(P_{5}\right),\left(P_{6}^{l}\right)$ and $\left(P_{7}^{l}\right)$ are fulfilled.

- Ifd is defined by: for $k=0, \ldots, N_{j}^{\prime}-1, d(k)=\delta_{k N}$, then $g_{j}$ is a right-sided $(N, j)^{\text {th }}$ order fractional modulating function on $[0, t]$, i.e. $\left(P_{5}\right),\left(P_{6}^{r}\right)$ and $\left(P_{7}^{r}\right)$ are fulfilled.

Proof. For $j \in\{0, \ldots, N-1\}$, we calculate ${ }_{s}^{C} \mathcal{D}_{\tau, t}^{j^{\prime} \alpha} g_{j}$ for $j^{\prime}=0, \ldots, N$, then by applying Lemma 4 we get: $\forall \tau \in[0, t]$,

$$
\begin{aligned}
& { }_{s}^{C} \mathcal{D}_{\tau, t}^{j^{\prime} \alpha} g_{j}(\tau)= \\
& \sum_{i=\sigma_{j^{\prime} j}} c_{i} \frac{\Gamma((i+j) \alpha+1)}{\Gamma\left(\left(i+j-j^{\prime}\right) \alpha+1\right)} t^{-i \alpha}(t-\tau)^{\left(i+j-j^{\prime}\right) \alpha},
\end{aligned}
$$

where $\sigma_{j^{\prime} j}=j^{\prime}-j$ if $j \leq j^{\prime}, \sigma_{j^{\prime} j}=0$, else. Since $i+$ $j-j^{\prime} \geq 0,\left(P_{5}\right)$ is fulfilled. Moreover, we have: for $j^{\prime}=$ $0, \ldots, \bar{N}-1$,

$$
{ }_{s}^{C} \mathcal{D}_{\tau, t}^{j^{\prime} \alpha} g_{j}(t)=\left\{\begin{array}{cl}
0 & \text { if } j^{\prime}<j, \\
c_{j^{\prime}-j} \Gamma\left(j^{\prime} \alpha+1\right) t^{\left(j-j^{\prime}\right) \alpha}, & \text { else. }
\end{array}\right.
$$

and

$$
\left[{ }_{s}^{C} \mathcal{D}_{\tau, t}^{j^{\prime} \alpha} g_{j}(\tau)\right]_{\tau=0}=t^{\left(j-j^{\prime}\right) \alpha} \sum_{i=\sigma_{j^{\prime} j}}^{N_{j}^{\prime}-1} c_{i} \frac{\Gamma((i+j) \alpha+1)}{\Gamma\left(\left(i+j-j^{\prime}\right) \alpha+1\right)}
$$

Since ${ }_{s}^{C} \mathcal{D}_{\tau, t}^{j^{\prime} \alpha} g_{j}(t)=0$ for $j^{\prime}<j$, if the coefficients $c_{i}$ are chosen such that $\left[{ }_{s}^{C} \mathcal{D}_{\tau, t}^{j^{\prime} \alpha} g_{j}(\tau)\right]_{\tau=0}=0$ for $j^{\prime}=$ 
$0, \ldots, N-1$, and ${ }_{s}^{C} \mathcal{D}_{\tau, t}^{j^{\prime} \alpha} g_{j}(t)=\delta_{j^{\prime} j}$ for $j^{\prime}=j, \ldots, N-1$, then $\left(P_{6}^{l}\right)$ and $\left(P_{7}^{l}\right)$ are fulfilled. For this, $N_{j}^{\prime}=2 N-j$ equations should be constructed. Hence, the linear system in (30) is obtained using (33) and (34), where $d(k)=$ $\delta_{k 0}$ for $k=0, \ldots, N_{j}^{\prime}-1$. Hence, if $c$ is the solution of this system, then $\left(P_{6}^{l}\right)$ and $\left(P_{7}^{l}\right)$ are fulfilled. Similarly, $\left(P_{6}^{r}\right)$ and $\left(P_{7}^{r}\right)$ can be fulfilled by solving the linear system in $(30)$, where $d(k)=\delta_{k N}$ for $k=0, \ldots, N_{j}^{\prime}-1$. Thus, this proof is completed.

It is important to remark that in Proposition 1, the matrix $Q$ and the vector $d$ in (30) are independent to $t$, so is the vector $c$. This is different from the construction proposed in [8], where a system needs to be solved for every $t \in I$.

\section{Simulation results}

In this section, numerical simulations are provided to demonstrate the efficiency of the proposed pseudo-state estimator. In the following example, the proposed estimator will be compared to the one obtained in [8].

Example: Let us take $N=4, \alpha=\frac{1}{3}, u=\sin (5 t)$, and the following matrices in (11)-(12): $B=(1,0,1,0)^{T}$, $C=\left(-\frac{14}{331}, \frac{98}{993}, \frac{14}{331},-\frac{34}{331}\right)$, and $A=\left(\begin{array}{cccc}1 & 0 & 0 & -2 \\ 3 & -3 & 0 & 3 \\ -1 & 1 & -5 & 4 \\ 1 & 0 & -1 & 0\end{array}\right)$, which satisfy conditions $\left(C_{1}\right)-\left(C_{3}\right)$. The pseudo-state $x$ of the system will be estimated on $I=[0,8]$ in discrete noisy case with a sampling period $T_{s}=0.0005$. Firstly, $x$ can be numerically obtained by using the Grünwald-Letnikov scheme [1]. Recall that the realstate of a fractional order system depends on both the pseudo-state and an initialization function which is also called history function $[6,25]$. Without considering the history function, the uniqueness of the state cannot be guaranteed. Consequently, the initialization problem is an important concept for fractional order systems [26]. In this example, by using a similar way as done in [7], the initialization of the considered fractional order system is realized as follows:

- the system is rest on $]-\infty,-3[$,

- the input $u=2 t$ is imposed to the system on $[-3,0]$,

- the history function of $x$ is generated on $[-3,0]$ by using Grünwald-Letnikov scheme with $x(-3)=$ $(0,0,0,0)^{T}$.

We get: $x(0)=(-0.8924,-2.1505,-1.1219,-1.0423)^{T}$. Then, the output $y$ is obtained on $I$ using $y=C x$. Let $y^{\varpi}\left(t_{i}\right)=y\left(t_{i}\right)+\sigma \varpi\left(t_{i}\right)$ be a discrete noisy observation of the output $y$ on $I$, where $t_{i}=i T_{s}$ for $i=0,1, \ldots, 10000$, $\left\{\varpi\left(t_{i}\right)\right\}$ is a zero-mean white Gaussian noise, and the value of $\sigma$ is adjusted such that the signal-to-noise ratio $\mathrm{SNR}=22 \mathrm{~dB}[27]$. It was shown in [8] that the modulating functions based pseudo-state estimator contains two sources of errors: the numerical errors due to a used numerical integration method and the noise error contribution due to the noisy output. Hence, so does the pseudo-state estimator proposed in this paper. In this example, the trapezoidal numerical integration method is applied to approximate the algebraic integral formulas for the estimator obtained in [8] and the one proposed in this paper. Recall that there is a design parameter $m$ in the estimator obtained in [8] to reduce the noise error. In order to give a guide to the choice of $m$, an error bound depending on $m$ was obtained for the noise error contribution. Consequently, by using this error bound analysis, the design parameter $m$ in the estimator obtained in [8] is chosen as: $m=2$ on [0,0.4[ and $m=14$ on $\left[0.4+T_{s}, 8\right]$. Finally, by applying Theorem 1 , Theorem 2 and Corollary 1 , the obtained estimation results for $x_{2}$ and the corresponding estimation errors are shown in Fig. 1 - Fig. 2. Remark that the estimation errors are mainly due to the noise error contribution. The simulation results clearly demonstrate the efficiency and robustness of the proposed pseudo-state estimator.

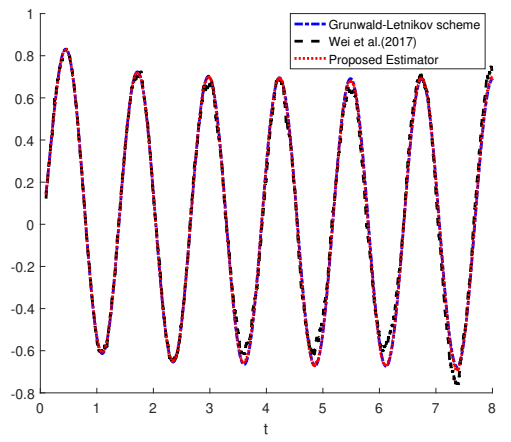

Fig. 1. Estimations of $x_{2}$.

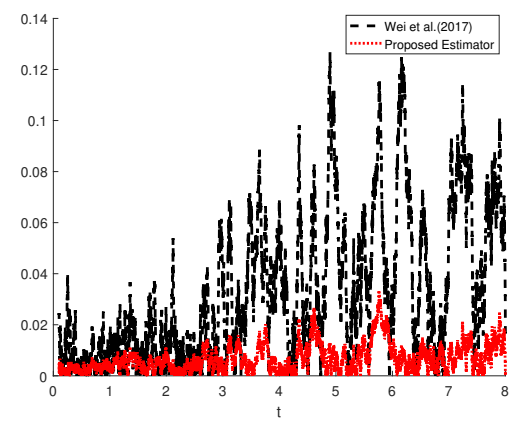

Fig. 2. Estimation errors of $x_{2}$.

\section{Conclusions}

In this paper, a non-asymptotic and robust pseudo-state estimator for a class of commensurate fractional order 
linear systems was designed in noisy environment. Compared to the pseudo-state estimator obtained in [8], the estimation process is greatly simplified due to the unnecessary of transforming the fractional sequential derivatives to the classical ones, which avoids the elimination of undesired terms and the generation of additional equations. By successively applying the fractional integration by parts formula, the required fractional derivatives and fractional order initial values were analogously obtained by algebraic integral formulas in continuous noise-free case. Moreover, different from [8] the time independence of the fractional modulating functions construction elegantly improves the efficiency of the proposed pseudostate estimator. Numerical results show the efficiency of the proposed pseudo-state estimator and the robustness with respect to noises is better than [8].

\section{Acknowledge}

The authors gratefully acknowledge the editors and reviewers for the valuable suggestions and helpful remarks to improve the quality our manuscript.

\section{References}

[1] Monje, C.A., Chen, Y.Q., Vinagre, B.M., Xue, D.Y., \& FeliuBatlle, V. (2010). Fractional-order Systems and Controls: Fundamentals and Applications. Springer.

[2] Liu, D.Y., Gibaru, O., Perruquetti W., \& Laleg-Kirati, T.M. (2015). Fractional order differentiation by integration and error analysis in noisy environment. IEEE Transactions on Automatic Control, 60(11), 2945-2960.

[3] Liu, D.Y., Zheng, G., Boutat, D., \& Liu, H.R. (2017). Non-asymptotic fractional order differentiator for a class of fractional order linear systems. Automatica, 78, 61-71.

[4] Cheng, S.S., Wei, Y.H., Chen, Y.Q., Wang, Y., \& Liang, Q. (2017). Fractional-order multivariable composite model reference adaptive control. International Journal of Adaptive Control and Signal Processing.

[5] Ahmed, S., Wang, H.P., \& Tian, Y. (2018). Model-Free Control Using Time Delay Estimation and Fractional-Order NonSingular Fast Terminal Sliding Mode for Uncertain Lower Limb Exoskeleton. Journal of Vibration and Control, DOI: $10.1177 / 1077546317750978$

[6] Sabatier, J., Farges, C., \& Trigeassou, J.C. (2014). Fractional systems state space description: some wrong ideas and proposed solutions. Journal of Vibration and Control, 20(7), 1076-1084.

[7] Belkhatir, Z., \& Laleg-Kirati, T. M. (2017). High-order sliding mode observer for fractional commensurate linear systems with unknown input. Automatica, 82, 209-217.

[8] Wei, X., Liu, D.Y., \& Boutat, D. (2017). Non-asymptotic state estimation for a class of fractional order linear systems. IEEE Transactions on Automatic Control, 62(3), 1150-1164.

[9] Kamal, S., Raman, A., \& Bandyopadhyay, B. (2013). FiniteTime Stabilization of Fractional Order Uncertain Chain of Integrator: An Integral Sliding Mode Approach. IEEE Trans. on Autom. Control, 58(6), 1597-1602.
[10] Bandyopadhyay, B., \& Kamal, S. (2015). Stabilization and control of fractional order systems: a sliding mode approach, Springer International Publishing.

[11] Shinbrot, M. (1954). On the analysis of linear and nonlinear dynamic systems from transient-response data, National Advisory Committee for Aeronautics NACA, Technical Note 3288, Washington.

[12] Co, T.B., \& Ungarala, S. (1997). Batch scheme recursive parameter estimation of continuous-time system using the modulating functions method. Automatica, 33(6), 1185-1191.

[13] Liu, D.Y., Laleg-Kirati, T.M., Gibaru, O., \& Perruquetti, W. (2013). Identification of fractional order systems using modulating functions method, in Proc. 2013 American Control Conference, Washington, DC, USA.

[14] Aldoghaither, A., Liu, D.Y., \& Laleg-Kirati, T.M. (2015). Modulating Functions Based Algorithm for the Estimation of the Coefficients and Differentiation Order for a Space Fractional Advection Dispersion Equation. SIAM Journal on Scientific Computing, 37(6), 2813-2839.

[15] Liu, D.Y., \& Laleg-Kirati, T.M. (2015). Robust fractional order differentiators using generalized modulating functions method. Signal Processing, 107, 395-406.

[16] Liu, D.Y., Tian, Y., Boutat, D., \& Laleg-Kirati, T.M. (2015). An algebraic fractional order differentiator for a class of signals satisfying a linear differential equation. Signal Processing, 116, 78-90.

[17] Fliess, M. (2006). Analyse non standard du bruit. C.R. Acad. Sci. Paris, Ser. I, 342, 797-802.

[18] Podlubny, I. (1999). Fractional Differential Equations. Academic Press, New York, NY, USA.

[19] Kilbas, A.A., Srivastava, H.M., \& Trujillo, J.J. (2006). Theory and Applications of Fractional Differential Equations, vol. 204 of North-Holland Mathematics Studies, Elsevier, Amsterdam, The Netherlands.

[20] Abramowitz, M., \& Stegun, I.A., \& editeurs (1965). Handbook of mathematical functions, GPO.

[21] Podlubny, I., \& Chen, Y.Q. (2007). Adjoint fractional differential expressions and operators, in Proc. the ASME 2007 International Design Engineering Technical Conferences 8 Computers and Information in Engineering Conference IDETC/CIE 2007, Las Vegas, NV, September 4-7.

[22] Petráš, I (2010). Fractional-Order Nonlinear Systems: Modeling, Analysis and Simulation. Springer.

[23] Pope, C (2008). Geometry and Group Theory, Texas A\&M University.

[24] Preising, H.A., \& Rippin, D.W.T. (1993) Theory and application of the modulating function method. I: Review and theory of the method and theory of the splinetype modulating functions. Computers and Chemical Engineering, 17, 1-16.

[25] Hartley, T.T., Lorenzo, C.F., Trigeassou, J., \& Maamri, N. (2013). Equivalence of history-function based and inifinitedimensional-state initializations for fractional-order operators. Journal of Computational and Nonlinear Dynamics, 8(4), 041014.

[26] Sabatier, J., Merveillaut, M., Malti, R., \& Oustaloup, A. (2010). How to impose physically coherent initial conditions to a fractional system? Commun Nonlinear Sci Numer Simulat, $15,1318-1326$.

[27] Haykin, S., \& Van Veen, B. (2002). Signals and Systems, 2nd edn. John Wiley \& Sons. 